\title{
VIRTUALIZING REAL OBJECTS WITH SURFACE INTERREFLECTIONS
}

\section{Takashi Machida}

\author{
Cybermedia Center, Osaka University \\ 1-30 Machikaneyama, Toyonaka, Osaka \\ E-mail: machida@ime.cmc.osaka-u.ac.jp
}

\author{
Naokazu Yokoya \\ Graduate School of Information Science, \\ Nara Institute of Science and Technology \\ 8916-5 Takayama, Ikoma, Nara \\ E-mail: yokoya@is.aist-nara.ac.jp
}

\begin{abstract}
In mixed reality, it is important to estimate object surface reflectance properties to render real objects under arbitrary illumination conditions. The authors developed a method to estimate reflectance properties of object surfaces densely. However, it was difficult to estimate surface reflectance properties faithfully for objects with interreflections. This paper describes a new method of densely estimating nonuniform surface reflectance properties of real objects constructed of convex and concave surfaces with interreflections. The proposed method first determines positions of light to take color images for discriminating diffuse and specular reflection components of surface reflection. Then, surface reflectance parameters are estimated based on radiosity. Experiments show the usefulness of the proposed method.
\end{abstract}

\section{INTRODUCTION}

In constructing a mixed reality environment, it is required to obtain surface reflectance properties of virtualized real objects. This could make it possible to merge virtualized objects seamlessly into a virtual world under arbitrary illumination conditions. For this purpose, we have conducted a research to estimate surface reflectance properties densely from range and surface texture images [1].

Object surface reflectance properties consist of diffuse and specular components. The diffuse reflection component is easily observed due to its nature of reflection in omnidirection. On the other hand, it is difficult to observe the specular reflection component due to its nature of reflection within a fixed angle with respect to a viewing position, light source and object surface normal vector. Moreover, even if the specular reflection component is observed, this component cannot be estimated stably due to small values. Our resent research has solved this problem by selecting optimum light positions to observe both components [1]. However, there still exists a problem such that interreflections are not considered.
In this paper, we take notice of the radiosity rendering method which can represent interreflections based on the transmission of radiosity (heat energy of light). Fournier et al. [2] has originally developed a method to estimate surface reflectance properties using the radiosity method in an indoor scene. This method assumes uniform reflectance on surface and determines only the diffuse reflectance parameter. There are some attempts to estimate both surface reflectance properties of a room $[3,4]$. They, however, also assume that almost all objects have the same reflectance properties, therefore, their algorithm cannot be applied to an object which has non-uniform surface reflectance properties.

This paper proposes a new method for estimating nonuniform reflectance properties of objects with interreflections to represent virtualized objects photo-realistically. The proposed method can observe the specular reflection component densely considering interreflections based on radiosity.

\section{ESTIMATION OF REFLECTANCE PARAMETERS FROM RANGE AND COLOR IMAGES}

Figure 1 shows a flow diagram of densely estimating surface reflectance properties of real 3D objects. Our process can be divided into three parts: Measurement of object $(A, C)$, selection of light source (B), and estimation of reflectance parameters (D).

\subsection{Measurement of object}

We use a laser rangefinder (Cyberware 3030RGB) with known positions of point light sources and a camera for acquiring surface color images, which is illustrated in Figure 2 (a). This system can obtain registered range and surface color texture images at the same time by rotating the rangefinder and the camera around an object, so that there is no registration error, even when the object is measured many times. 


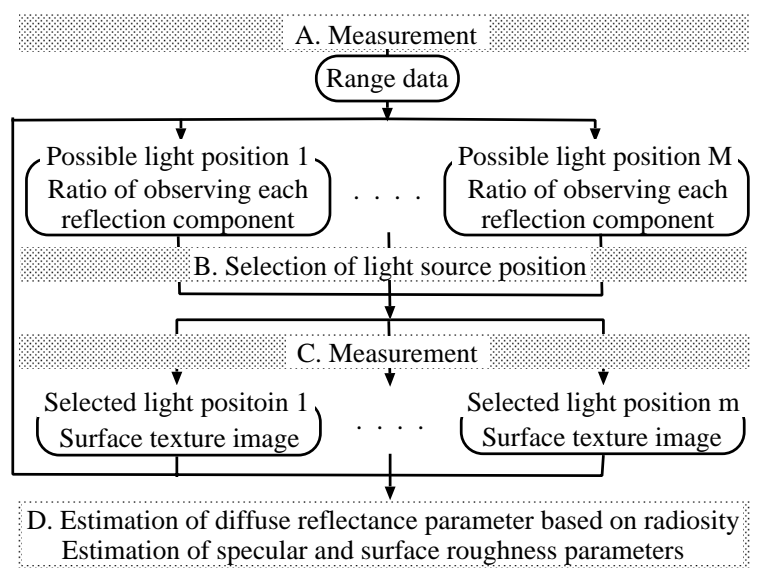

Figure 1: Flow diagram of estimating surface reflectance properties.

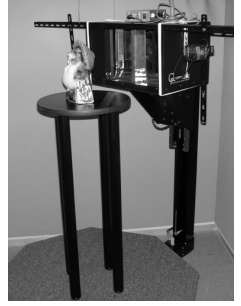

(a) Appearance of 3D-Digitizer

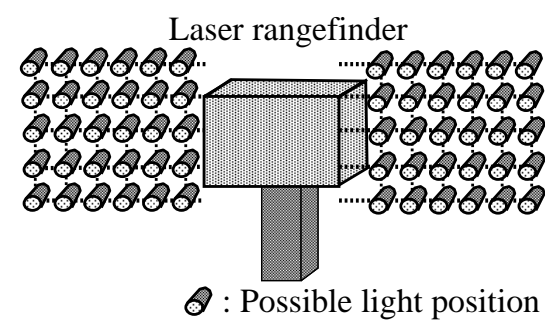

(b) 60 possible light positions
Figure 2: 3D-Digitizer and experimental setup.

\subsection{Selection of positions of light source}

In the present experimental setup, multiple positions of a light are determined among 60 possible positions prepared around the laser rangefinder and these are twodimensionally arranged at the interval of $5 \mathrm{~cm}$ as shown in Figure 2 (b). The positions of a camera and a light source are calibrated in advance. When optimum light positions are selected, a single light is attached at the selected positions in turn. Therefore, the calibration of brightness among multiple lights is not needed.

Here, we employ the Torrance-Sparrow model [5] to represent object reflectance properties physically. The Torrance-Sparrow model is given as:

$$
i=\frac{Y}{D^{2}}\left\{P_{d} \cos \theta_{d}+\frac{P_{s}}{\cos \theta_{v}} \exp \left(-\frac{\theta_{r}^{2}}{2 \sigma^{2}}\right)\right\},
$$

where $i$ represents an observed intensity, $D$ is an attenuation coefficient concerning the distance between a point light source and an object surface point, $Y$ represents the strength of a light source. $P_{d}, P_{s}$ and $\sigma$ are the diffuse reflectance, the specular reflectance and the surface roughness parameters, respectively. $\theta_{d}$ is an angle between a light source vector and a surface normal vector, $\theta_{v}$ is an angle between a viewing vector and a surface normal vector, and $\theta_{r}$ is an angle between a viewing vector and a reflection vec- tor. Note that the reflection vector is a vector to which a light vector is mirrored against a normal vector.

It is required to observe each surface point under three different lighting conditions: One for determining $P_{d}$ and others for acquiring $P_{s}$ and $\sigma$. Here, if the value of $\theta_{r}$ is small, the specular reflection component is judged to be reduced seriously. Therefore, we can use the value of $\theta_{r}$ to judge whether the specular reflection component is observed or not. The selection of position of a light source is repeated until almost all surface points satisfies three different lighting conditions (See [1] for detail). Then, a certain number of light positions, say $m$, are selected to densely observe both reflection components.

\subsection{Estimation of reflectance parameters}

A texture image is obtained with a selected light position $p(p=1, \cdots, m)$ and consists of $n$ pixels $\left(i_{p 1}, \cdots, i_{p n}\right)$, where $i_{p k}$ means a color intensity. Each pixel is classified into three types $T_{\text {diff. }}, T_{\text {spec }}$ and $T_{n o n e} . T_{\text {diff. }}$ means a pixel containing only the diffuse reflection component and $T_{\text {spec }}$ means a pixel containing strong specular reflection component. $T_{\text {none }}$ means a pixel classified into neither $T_{\text {diff }}$ nor $T_{\text {spec }}$ and such a pixel is not used to estimate reflectance parameters.

\subsubsection{Estimation of diffuse reflectance parameter based on radiosity}

We assume in this paper that interreflections do not have an influence from both reflection components but only from the diffuse reflection component. Therefore, a pixel which is categorized into $T_{\text {diff } f}$ is used in the following method. In this paper, to consider interreflections, we employ the radiosity equation given as:

$$
B_{u}=E_{u}+P_{d u} \sum_{v=1}^{\alpha} B_{v} F_{u v},
$$

where $B_{u}$ and $B_{v}(1 \leq u, v \leq \alpha)$ represent the radiosity of $u$-th and $v$-th patch, respectively. Note that $\alpha$ is the number of object patches. $F_{u v}$ is a form factor between the patch $u$ and the patch $v . E_{u}$ is an environment term which represents an influence on the patch $u$. In this paper, we assume that $E_{u}=0$, because the object is measured in a dark room. $P_{d u}$ represents the diffuse reflectance parameter on the patch $u$.

In the proposed method, the form factor $F_{u v}$ is known because the object shape is measured by the laser rangefinder. Since the range and texture images are registered at each surface point, the radiosity $B_{u}$ of the patch $u$ is calculated based on the value of the surface point which corresponds to the patch $u$. Then, the diffuse reflectance parameter $P_{d u}$ is estimated by solving Eq. (2). Finally, the diffuse reflectance 


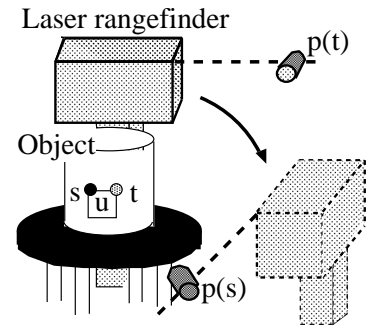

Figure 3: Calculation of radiosity.

parameter at a each surface point is estimated by calculating the average among neighbouring patches which share the point.

Here, the calculation of radiosity $B_{u}$ is described. Let $p(x)$ be the position of a light source when a surface point $x$ is measured as shown in Figure 3, and $e_{x}$ be the value of the surface point $x$. The radiosity $B_{u}$ of the patch $u$ which includes surface points $s$ and $t$ is calculated as follows. Each patch consists of four points and $B_{u}$ is represented as the sum of the values of the corresponding pixels. Because a light source attached with the laser rangefinder moves during measurement, it is necessary to calculate $e_{t}$ with the light position $p(s) . \quad e_{t}$ is interpolated linearly by the following equation since $e_{t}$ and $e_{s}$ are categorized in $T_{\text {diff } f \text {. }}$.

$$
e_{t}=\frac{\mathbf{N}_{t} \cdot \mathbf{L}_{p(s) t}}{\mathbf{N}_{s} \cdot \mathbf{L}_{p(s) s}} e_{s},
$$

where $\mathbf{N}_{s}$ and $\mathbf{N}_{t}$ are normal vectors at surface points $s$ and $t . \mathbf{L}_{p(s) s}$ and $\mathbf{L}_{p(s) t}$ are light vectors with the light position $p(s)$ at surface points $s$ and $t$. Note that in the case of $\mathbf{N}$. $\mathbf{L} \leq 0, e_{t}$ should be 0 to consider the measurability of light reflection.

\subsubsection{Estimation of specular reflectance and surface roughness parameters based on Torrance-Sparrow model}

The specular reflectance parameter $P_{s k}$ and the surface roughness parameter $\sigma_{k}$ at the $k$-th pixel which is categorized in $T_{\text {spec }}$ are estimated by solving a simultaneous equation of Eq. (1) with the values of the specular reflection component extracted from two images taken under two different illumination conditions and $P_{d k}$ estimated above. See [1] for detail. Note that $P_{d k}$ estimated above should be scaled to Eq. (1) before computing the specular and surface roughness parameters.

\section{EXPERIMENTS}

We show that the proposed method can remove an influence of interreflections in a preliminary experiment. We separate the object A shown in Figure 4 (a) into two regions $\mathrm{A}$ and $\mathrm{B}$. We have conducted two preliminary experiments. One is that the same white paper with a uniform diffuse reflectance is pasted up on both regions. The other is that the

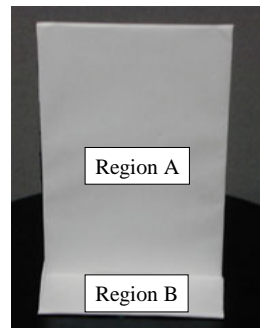

(a) Object $\mathrm{A}$

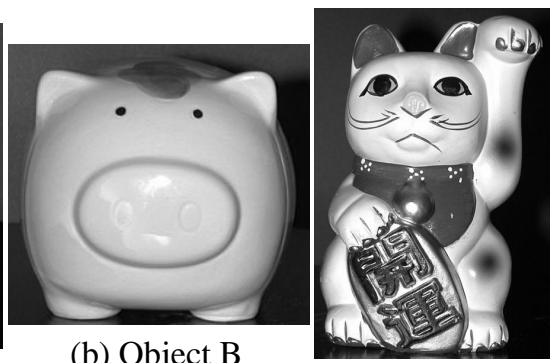

(c) Object C
Figure 4: Three objects used in experiment.

Table1: Measurability of two reflection components.

\begin{tabular}{|c|c|c|}
\hline Measurment object & B & C \\
\hline The number of selected light positions & $9(3)$ & $13(5)$ \\
\hline Diffuse reflection component & $100.0 \%$ & $100.0 \%$ \\
\hline Specular reflection component & $94.7 \%$ & $85.9 \%$ \\
\hline
\end{tabular}

same glossy paper with a uniform reflectance is pasted up on both regions. The proposed method is compared with the conventional method [1]. Results are shown in Figures 5 and 6. Each graph represents RGB channels of the diffuse reflectance parameter estimated by both methods. The horizontal axis means the position of the surface point in the vertical direction of the object and the vertical axis means the average of diffuse reflectance parameters in the horizontal direction of the object. In Figure 5, in the conventional method, the value of the diffuse reflectance parameter is large around the boundary between the regions A and $\mathrm{B}$ because the conventional method does not consider interreflections. On the other hand, such an effect is eliminated in the proposed method. This clearly shows the usefulness of the proposed method. However, the influence of interreflections still remains in the glossy surface as shown in Figure 6, because interreflections due to the specular reflection also ocurrs at the boundary between the regions $\mathrm{A}$ and $\mathrm{B}$ in the second preliminary experiment.

In the next experiment, We show that the proposed method is useful for real objects with complex shapes: Objects $B$ and $C$ shown in Figures 4 (b) and (c). The measurability of both reflection components and the number of selected light sources are shown in Table 1. The number in the bracket means the number of selected positions of a light source required to estimate the diffuse reflectance parameter. From these results, it is observed that non-uniform surface reflectance properties are estimated over almost the whole surface considering interreflections with a limited number of light positions.

Figure 7 shows rendering results of virtualized objects B and $\mathrm{C}$ with multiple viewing angles, respectively. A virtual light source is located at a fixed position. In rendering, direct reflection from the virtual light source is represented by using the Torrance-Sparrow model. Each object is rendered suitably even at the part which contains interreflections due to the diffuse reflection component. However, in the case 


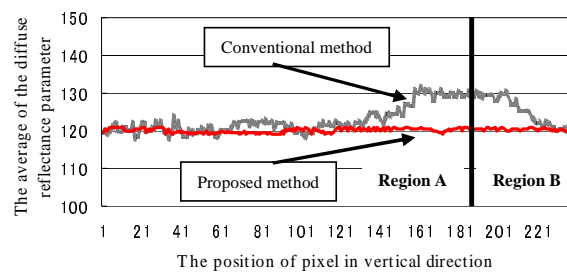

(a) R channel

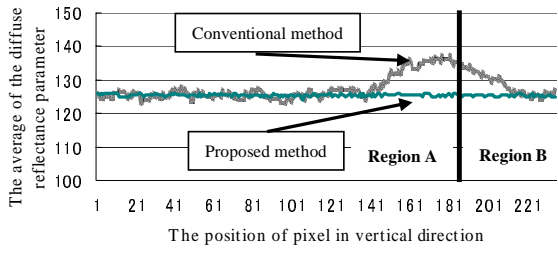

(b) G channel

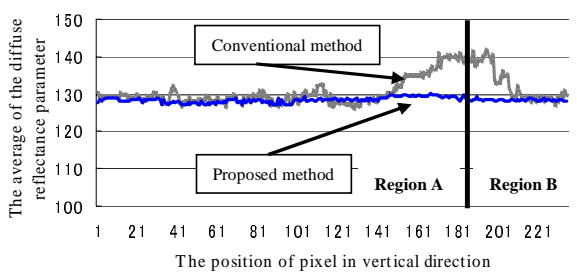

(c) B channel

Figure 5: A comparison with previous work for a diffuse surface.

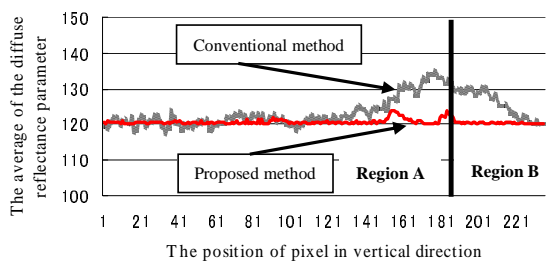

(a) R channel

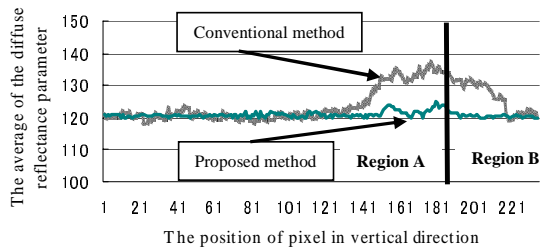

(b) G channel

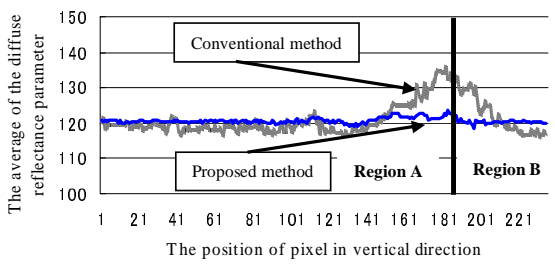

(c) B channel

Figure 6: A comparison with previous work for a glossy surface.

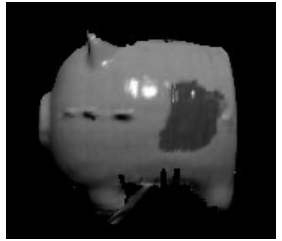

Left

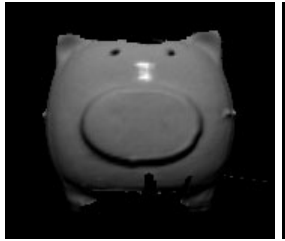

Front

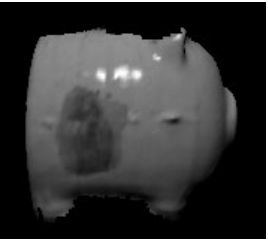

Right

(a) Object B

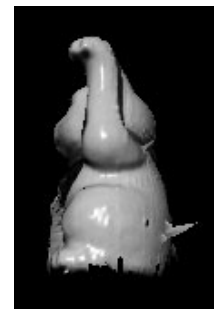

Left

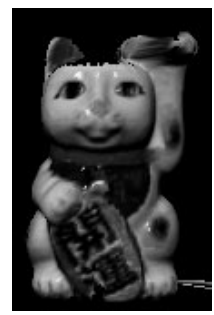

Front

(b) Object C

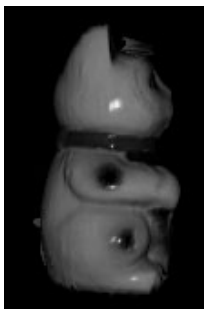

Right

Figure 7: Rendering of virtualized objects with Torrance-Sparrow model.

of object $\mathrm{C}$ which has a complicated shape, interreflections due to the specular reflection component cause some errors. It is necessary to eliminate such an influence.

\section{CONCLUSIONS}

In this paper, we have proposed a new method of densely estimating non-uniform reflectance properties of real objects with interreflections. In the proposed approach, we have estimated surface reflectance properties based on radiosity. The experiments have shown that the proposed method is useful for estimating the reflectance parameters of objects which exhibit non-uniform surface reflectance. Some extensions for considering specular interreflections should further be investigated.

\section{ACKNOWLEDGMENTS}

This work was supported in part by Grant-in-Aid for Scientific Research under Grant No. 13480098 from the Ministry of Education, Culture, Sports, Science and Technology.

\section{REFERENCES}

[1] T. Machida, H. Takemura, and N. Yokoya, "Dense estimation of surface reflectance properties for merging virtualized objects into real images," Proc. ACCV 2002, pp. 688-693, January 2002.

[2] A.Fournier, A.S. Gunawan, and C. Romanzin, "Interactive common illumination for computer augmented reality," Proc. Graphics Interface 1993, pp. 254-262, May 1993.

[3] C. Loscos, G. Drettakis, and L. Robert, "Interactive virtual relighting of real scenes," IEEE Trans. Visualization and Computer Graphics, vol. 6, no. 4, pp. 289-305, December 2000.

[4] Y. Yu, P. E. Debevec, J. Malik, and T. Hawkins, "Inverse global illumination: Recovering reflectance models of real scenes from photographs," Proc. SIGGRAPH '99, pp. 215-227, August 1999.

[5] K. E. Torrance and E. M. Sparrow, "Theory for offspecular reflection from roughened surfaces," Journal of Optical Society of America, pp. 1105-1114, 1967. 